\title{
Piomiositis en un adulto con diabetes mellitus
}

\author{
Pyomyositis in an adult with diabetes mellitus
}

\author{
Enrique Otoya'
}

Otoya E. Piomiositis en un adulto con diabetes mellitus. Rev Soc Peru Med Interna. 2021;34(4):177-I80.DOI: https://doi.org/10.36393/ spmi.v34i4.634

\begin{abstract}
RESUMEN
Paciente varón de 63 años de edad, diabético y en tratamiento con insulina, con dolor y aumento de volumen a nivel del hemitórax anterior izquierdo, con estudio de ecpgrafía y tomografía compatible con colección a nivel del músculo pectoral mayor. En la punción se obtuvo un material purulento y fétido, confirmándose el diagnóstico de piomiositis. Se aisló Streptococcus constellatus en el cultivo del pus. Con pobre respuesta a tratamiento médico inicial, requirió drenaje quirúrgico y cobertura antibiótica amplia
\end{abstract}

Palabras claves: piomiositis, diabetes mellitus, Streptococcus constellatus.

\section{ABSTRACT}

A 63-year-old male patient, diabetic and on insulin treatment, with pain and increased volume at the level of the left anterior hemithorax, with ultrasound and tomography study compatible with collection at the level of the pectoralis major muscle. In the puncture a purulent and fetid material was obtained, confirming the diagnosis of pyomyositis. Streptococcus constellatus was isolated in pus culture. With poor response to initial medical treatment, it required surgical drainage and broad antibiotic coverage.

Keywords: pyomyositis, diabetes mellitus, Streptococcus constellatus.

\section{INTRODUCCIÓN}

La piomiositis primaria es la infección bacteriana del músculo estriado con formación de abscesos, ocasionada por diseminación hematógena del agente causal, no asociada con diseminación por contigüidad de piel, partes blandas ni hueso. ${ }^{1,2}$ Se relaciona con condiciones que deterioran los mecanismos generales de defensa del huésped, como HIV/sida, diabetes, desnutrición, neoplasias, enfermedades autoinmunes, hepatopatías crónicas, drogadicción endovenosa, uso de esteroides,

I Médico internista. Servicio de Medicina Interna, Hospital EsSalud Guillermo Kaelin de la Fuente, Villa María del Triunfo, Lima, Perú. etc., pudiendo asociarse a factores locales, como injurias musculares, microtraumatismos o estrés muscular.

Los músculos comúnmente afectados son los grandes grupos musculares de la cintura pelviana y de miembros inferiores, pudiendo ser unifocal o multifocal. Los sitios menos afectados son la pared abdominal, pared torácica y las regiones paraespinales. ${ }^{3}$

Según la serie de Bickels y col., en 452 aislamientos en pacientes con piomiositis primaria, el principal agente etiológico fue Staphylococcus aureus (77\%), siguiendo en frecuencia estreptococos (11\%), Escherichia coli $(2,4 \%)$ $\mathrm{y}$, menor al 2\%, Salmonella enteritidis, Mycobacterium tuberculosis, Streptococcus pneumoniae. Otros microorganismos involucrados en casos aislados han sido Klebsiella pneumoniae, Yersinia enterocolitica, Neiseria 
gonorrhoeae, Aeromonas hydrophila, anaerobios y hongos. El cuadro clínico se caracteriza por fiebre, síntomas locales como dolor, tumefacción y aumento de tamaño del músculo. Para el diagnóstico se requiere de la sospecha clínica y del aporte de las imágenes. ${ }^{4}$ Las anormalidades en el laboratorio difícilmente colaboran en establecer el diagnóstico, por lo que el reconocimiento temprano como el tratamiento oportuno son las claves para evitar cuadros graves.

\section{PRESENTACIÓN DEL CASO}

El caso corresponde a un varón de 63 años, natural y procedente de Lima, con antecedentes de hipertensión arterial, diabetes mellitus con insulinoterapia y con controles irregulares de glucosa. Ingresó por primera vez al servicio de emergencia por presentar 10 días de dolor torácico anterior, entre las regiones esternal y pectoral mayor izquierda, sin antecedente de trauma, rascado y/o herida en la zona. Al examen físico de ingreso, se evidenció aumento de volumen y dolor a la palpación a nivel de tórax anterior izquierdo, sin eritema ni calor en la piel suprayacente. Recibió analgésicos parenterales con mejoría parcial. Se solicitó una ecografía de partes blandas, la cual reportó signos ecográficos compatibles con absceso en formación en partes blandas de la pared torácica (Figura 1). Se indicó clindamicina $300 \mathrm{mg}$ cada 8 horas por 7 días e Ibuprofeno $400 \mathrm{mg}$ cada 12 horas por 5 días y se le dio de alta.

Cuatro días después retornó por persistencia del dolor, que se extendía al miembro superior izquierdo, asociado a malestar general y escalofríos. Al examen, se objetivó dolor a la palpación, aumento de volumen a nivel esternal, de consistencia renitente y eritema. Se decidió drenaje de la colección a nivel esternal, obteniendo secreción purulenta, muy fétida, aprox. $100 \mathrm{cc}$, se tomó muestra para cultivo, se dejó un dren y se hospitalizó con diagnóstico de absceso de pared torácica, iniciándose ciprofloxacina más clindamicina por vía endovenosa.

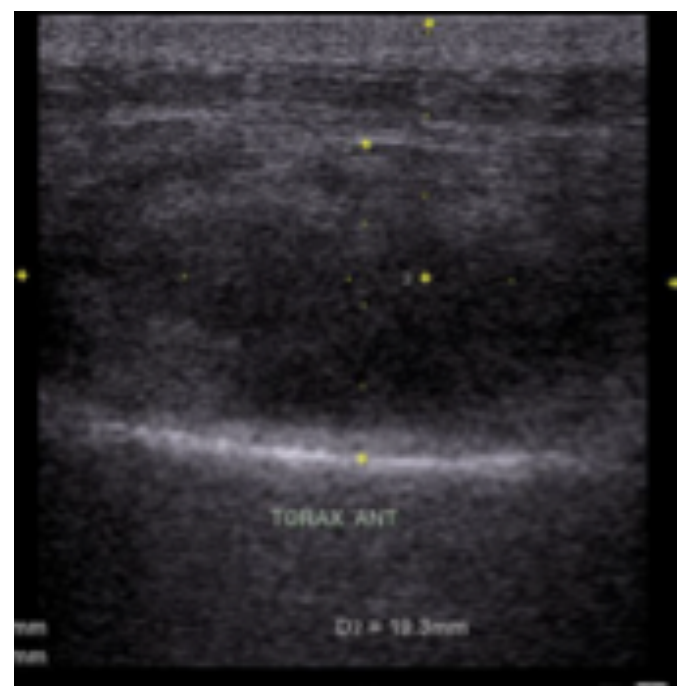

Figura I. Ecografía: colección en la región pectoral izquierda.

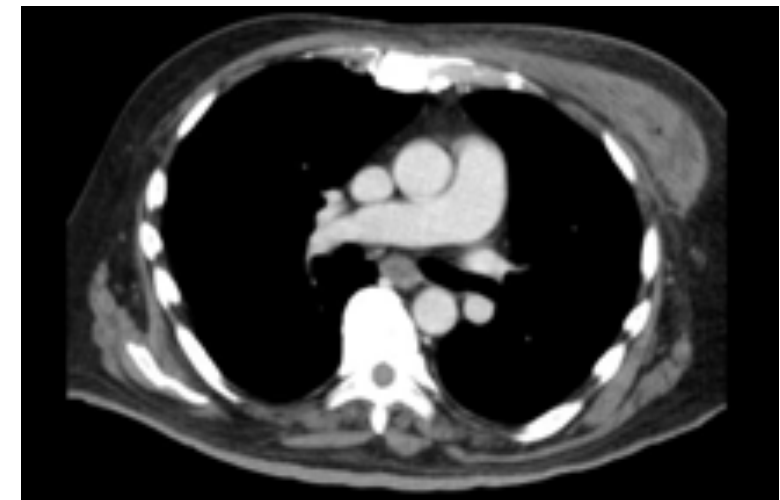

Figura 2. Día I de hospitalización - día I 5 de enfermedad. colección densa pectoral izquierda.

En los exámenes iniciales de ingreso se evidenció hemoglobina 12,8 g/dl, plaquetas 385 mil, leucocitos 23700 células $/ \mathrm{mm}^{3}$ con 20140 segmentados (13\% abastonados) y 2130 linfocitos; glucosa basal $158 \mathrm{mg} / \mathrm{dl}$, creatinina 0,79 $\mathrm{mg} / \mathrm{dl}$, urea $60 \mathrm{mg} / \mathrm{dl}$, PCR $32 \mathrm{mg} / \mathrm{dl}$.

Se evidenció secreción purulenta por el drenaje a nivel del esternón y nuevo aumento de volumen de consistencia renitente con eritema a nivel pectoral izquierdo, por lo que se realiza tomografía de tórax que mostró una colección densa a nivel del pectoral izquierdo de volumen aprox. 60cc (Figura 2). El cultivo de secreción del primer drenaje $\mathrm{y}$ dos hemocultivos fueron negativos. Ante la progresión clínica, se decidío un nuevo drenaje a nivel del pectoral mayor, obteniéndose 150 cc de secreción "achocolatada" y se tomaron nuevos cultivos.

Durante su cuarto día de estancia hospitalaria se notó extensión de edema a cara anterior del brazo izquierdo, con empastamiento y aumento de volumen a lo largo del miembro superior izquierdo, en forma homogénea. La tomografía de tórax control y con extensión al miembro superior izquierdo (Figuras 3a y 3b) evidenció una colección de contenido líquido y aéreo a nivel del pectoral mayor izquierdo, aprox. $200 \mathrm{cc}$, que se extendía a la región esternal y axilar, con volumen aprox. de $100 \mathrm{cc}$, comprometiendo también al tejido celular subcutáneo de la cara interna del brazo hasta el codo, adyacente a la fascia muscular con volumen de $60 \mathrm{cc}$. y densidades aéreas hasta el tercio proximal del brazo. A nivel de antebrazo se observó una colección laminar. El derrame pleural bilateral observado en la tomografía fue atribuido a falla cardiaca por sobrehidratación, lo cual se corrigió en los días siguientes. Se cambió la cobertura antibiótica con vancomicina 1 gramo cada 12 horas y meropenem 1 gramo cada 8 horas. Sin embargo, el paciente persistió con dolor a lo largo de región torácica anterior izquierda, extendiéndose a la región axilar y miembro superior izquierdo ipsilateral, drenajes de esternón y pectoral mayor con abundante secreción purulenta fétida, los exámenes de laboratorio control con persistencia de leucocitosis con desviación izquierda y PCR incrementado, sin mejoría a las 72 horas de haber cambiado de antibióticos. Debido a la mala evolución clínica y 

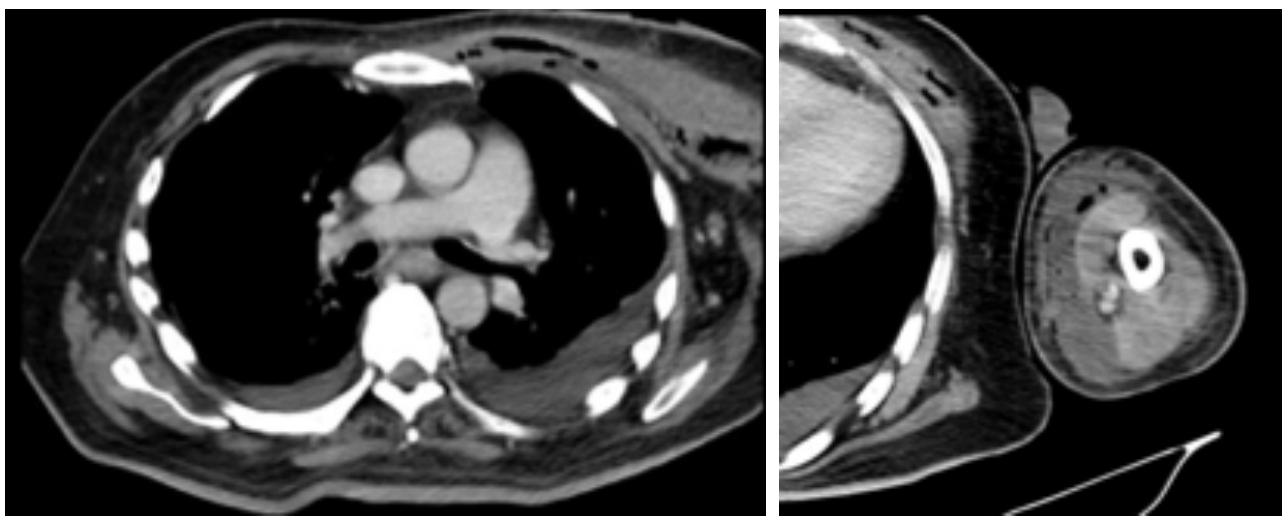

Figuras 3a y 3b. Día 4 de hospitalización - Día 19 de enfermedad.

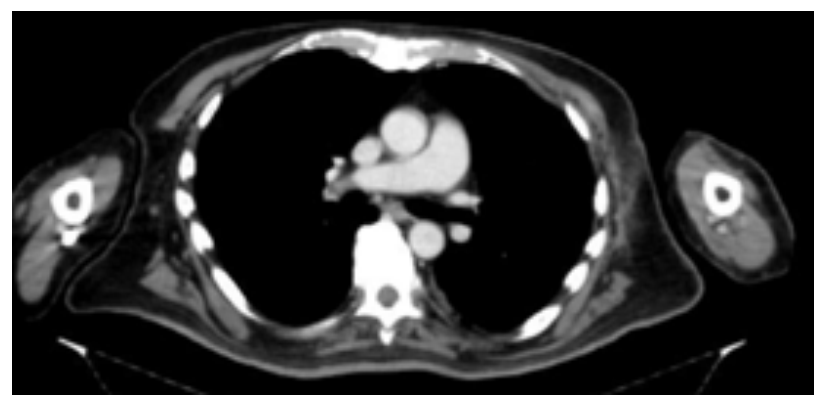

Figura 4. Día 30 de hospitalización - Día 45 de enfermedad.

laboratorial, se decidió el ingreso a sala de operaciones para debridamiento y colocación de nuevo drenaje a nivel axilar. Los hallazgos operatorios reportaron abundante colección purulenta a nivel del músculo pectoral mayor izquierdo, con extensión a la región infraclavicular, esternal, hueco axilar y hombro izquierdo, adenopatías axilares sin alteraciones macroscópicas, se drenó $400 \mathrm{cc}$ de líquido purulento que se envió a estudio y se colocó nuevo dren a nivel axilar, manteniendo la cobertura antibiótica instaurada.

En los días siguientes, el paciente tuvo una lenta evolución favorable, menor dolor, menor edema, disminución progresiva del volumen de drenaje, manteniendo características purulentas fétidas. Se cultivó Streptococcus constellatus de la secreción intraoperatoria del pectoral mayor izquierdo. Habiendo completado 14 días de meropenem y vancomicina, de acuerdo con el antibiograma, se pasó a ceftriaxona endovenosa. Se realizo tomografía de tórax control (Figura 4) evidenciando disminución significativa de la colección a nivel de pectoral y axilar, y la presencia de densidades aéreas laminares. Concomitantemente, hubo disminución progresiva de volumen en los drenajes, retirándose los drenes, primero el esternal, luego el pectoral y por último el axilar izquierdo. Salió de alta con disminución completa del edema en el miembro superior izquierdo, sin dolor, con tratamiento antibiótico vía oral seguimiento ambulatorio.

\section{DISCUSIÓN}

La piomositis, es la infección aguda del musculo estriado, relacionado con factores de riesgo que condicionan inmunosupresión. Nuestro paciente tenía el antecedente de diabetes mellitus insulino requiriente, lo que lo hacía más propenso a piomiositis que individuos no diabéticos. La diabetes mellitus puede propiciar daño muscular, lo que eventualmente incrementa el riesgo de infarto, miositis y piomiositis. Adicionalmente, los pacientes con diabetes mellitus son más susceptibles a microangiopatía lo que puede conllevar a insuficiencia vascular y alteraciones de la migración de neutrófilos al musculo. Asimismo, los pacientes con diabetes mellitus tienen un riesgo incrementado de colonización por Staph. aureus. ${ }^{5,6}$

En los estadios iniciales, cuando los músculos afectados son profundos, la aponeurosis muscular y la fascia profunda retardan transitoriamente el compromiso del tejido celular subcutáneo y la piel, y los signos inflamatorios superficiales suelen estar ausentes. Se pueden distinguir tres estadios. E1 primero, o estadio invasivo, se caracteriza por molestia muscular, con leve edema, fiebre baja y decaimiento, sin presencia de colección. Dado que sólo la aponeurosis se encuentra inervada, el dolor sobre el músculo demora en aparecer entre una y dos semanas, luego se produce la conformación del absceso. El segundo estadio, o purulento, es por la conformación del absceso, comienza a ser sintomático con dolor severo, rigidez muscular, edema moderado y fiebre; así, a la mayoría de los pacientes se los reconoce en este momento evolutivo, el cual se desarrolla entre los 10 a 21 días de iniciado los síntomas. De no ser tratado, la enfermedad evoluciona a un tercer estadio, el estadio de sepsis, donde presenta destrucción muscular, extensión local con osteomielitis y septicemia. Se han descrito casos de síndrome de choque tóxico debido a piomiositis. ${ }^{7,8}$

La presentación clínica de la piomiositis puede similar un cuadro de fascí́tis necrotizante e incluso pueden coexistir en pacientes inmunocomprometidos. ${ }^{9,10}$

Al examen físico no se encuentran adenopatías regionales. Inicialmente no es posible palpar el absceso dado que se encuentra en la profundidad del músculo, pero el área se encuentra firme, asociada con dolor y rigidez.

En los exámenes de laboratorio puede presentarse leucocitosis y eritrosedimentación elevada. Las concentraciones plasmáticas de creatinafosfoquinasa, 
aldolasa y aspartato aminotransferasa suelen ser normales a pesar de que se produzca una extensa necrosis muscular, lo cual permite la exclusión de miositis de otras etiologías. Los hemocultivos son positivos en $5 \%$ a $30 \%$ de los casos. ${ }^{11}$ El Streptococcus constellatus es parte de la flora microbiana de la vía aérea superior, puede aislarse en muestras de región faríngea, orofaríngea y región gingival. También puede encontrarse en el tracto gastrointestinal y en la región vaginal. Se le conoce por su facilidad para producir colecciones purulentas en partes blandas, infecciones intraabdominales, infecciones pulmonares, bacteriemia, infección del sistema nervioso central y endocarditis. Los casos de bacteriemia normalmente son atribuidos a focos gastrointestinales y/o del tracto respiratorio superior. ${ }^{12,13}$ Dentro de los estudios de imágenes para el apoyo diagnóstico, la ecografía y la tomografía son los exámenes iniciales para evaluar piomiositis y definir su estadio. La ecografía permite detectar en forma inmediata los focos de supuración intramusculares, evaluar el plano de la lesión y su dimensión. Su utilidad es mayor en la etapa supurativa y como guía para la punción diagnóstica y/o evacuatoria. Contribuye al diagnóstico diferencial con desgarros musculares, celulitis, colecciones y fascitis. La tomografía computarizada permite evaluar los planos de lesión y es útil para el estudio de los músculos profundos. La resonancia magnética, es considerada el gold standard, incluso en estadios iniciales, facilita la discriminación de los planos fasciales y el diagnóstico diferencial con fasciítis necrotizante. Su dificultad radica en el alto costo y baja accesibilidad. ${ }^{14}$

El tratamiento de la piomiositis dependerá del estadio en el que se encuentre. En el estadio inicial, el tratamiento antibiótico es suficiente, debiendo incluir una cobertura contra Staph. aureus. En los siguientes estadios, cuando el absceso ya está conformado, está indicado el drenaje percutáneo o desbridamiento quirúrgico asociado al tratamiento antibiótico de amplio espectro y será modificado subsecuentemente en base a la sensibilidad antibiótica del germen aislado. ${ }^{15,16}$

La duración del tratamiento no se encuentra establecida, generalmente se aplica durante tres a cuatro semanas, con terapéutica endovenosa inicial, pero puede variar considerablemente de acuerdo con la extensión de la enfermedad. El pronóstico es excelente, con la excepción de los casos diagnosticados en estadios avanzados, que cursan con choque séptico y fallo multiorgánico. La mortalidad varía entre $0,89 \%$ y $10 \%$ según diferentes series, siendo la sepsis la principal causa de muerte. ${ }^{17-19}$

Nuestro paciente se presentó clínicamente en un estadio 2, los exámenes de laboratorio descartaron miopatías y otras causas de inmunosupresión. La tomografía de tórax ayudo a encaminar el diagnostico. Sin embargo, si bien se inició cobertura antibiótica desde un inicio, dada la extensión del compromiso, requirió cobertura antibiótica amplia, empírica e ingreso a sala de operaciones para drenaje de la colección y colocación de dren, cursando con mejora paulatina.
Hemos querido dar a conocer este caso por lo infrecuente del diagnóstico, la localización inusual de la piomiositis, el poco apoyo de los exámenes auxiliares para el diagnóstico, por lo que se requiere la sospecha clínica y la interpretación adecuada de los exámenes de imágenes, para con ello, identificar el estadio de enfermedad y dar un tratamiento oportuno.

\section{REFRENCIAS BIBLIOGRÁFICAS}

I. Burdette SD, Watkins RR, Wong KK et al. Staphylococcus aureus pyomyositis compared with non-Staphylococcus aureus pyomyositis. J Infect. 2012;64:507-I2.

2. Chen WS, Wan YL. Iliacus pyomyositis mimicking septic arthritis of the hip joint. Arch Orthop Trauma Surg. 1996; I I5:233-5

3. Hall RL, Callghan JJ, Moloney E, Martínez S, Harrelson J. Pyomyositis in a temperate climate. Presentation, diagnosis, and treatment.J Bone Joint Surg Am. 1990; 72: 1240-4.

4. Saeed K, Esposito S, Gould I, Ascione T, Bassetti M, Bonnet E et al. Hot topics in necrotising skin and soft tissue infections. Int J Antimicrob Agents. 2018;52:I - I0.

5. Pozzilli P, Leslie R. Infections and diabetes: mechanisms and prospects for prevention. Diabet Med. 1994; I I:935-941.

6. Walling DM, Kaelin WG. Pyomyositis in patients with diabetes mellitus. Rev Infect Dis. 1991; 13:797-802.

7. Villamil-Cajoto I, Maceriras-Pan F, Villacián-Vicedo M. Piomiositis: presentación de 17 casos en niños y adultos. Rev Med Chile. 2006; I34: $3 \mid-4$.

8. Crum NF. Bacterial pyomyositis in the United States. Am J Med. 2004; I I 7: 420-8

9. Low DE, Norrby-Teglund A. Myositis, pyomyositis, and necrotizing fasciitis. Principles and Practice of Pediatric Infectious Diseases. Elsevier, London, 20I2, pp. 462-468.

10. Crum-Cianfl NF. Infectious myositis. Best Pract Res Clin Rheumatol. 2006;20:1083-96.

II. Stevens DL, Bisno AL, Chambers HF, Everett ED, Dellinger P, Goldstein EJ et al. Guidelines for skin and soft-tissue infections. Clin Infect Dis. 2005;4I:I373-406

12. Bickels J, Ben-Sira L, Kessler A, Weintroub S. Primary pyomyositis. J Bone Joint Surg. 2002; 84-A: 2277-86.

13. Koneman. Color Atlas and Textbook of Diagnostic Microbiology. 2017; | 3:765-6.

14. Gordon BA, Martinez S, Collins AJ. Pyomyositis: characteristics at CT and MR imaging. Radiology. 1995; 197:279-86.

I5. Hall RL, Callaghan JJ, Maloney E, Martinez S, Harrelson JM. Pyomyositis in a temperate climate. Presentation, diagnosis, and treatment.J Bone Jt Surg. 1990;72:1240-44.

16. Kwak YG, Choi SH, Kim T, Park SY, Seo SH, Kim MB et al. Clinical guidelines for the antibiotic treatment for community-acquired skin and soft tissue infection. Infect Chemother. 2017;49:30I-325.

17. Back SA, Fishbein G, Gwinup G. A case of group B streptococcal pyomyositis. Rev Infect Dis. 1990; 12:784-7.

18. Adams EM, Gudmundsson S, Yocum DE, Haselby RC, Craig WA, Sundstrom WR. Streptococcal myositis. Arch Intern Med. 1985; 145: 1020-3

19. Consenso SADI-SAM-SAD-CACCVE. Guía para el manejo racional de las infecciones de piel y partes blandas. Rev Panam Infectol. 2009; I I: 50-5.

\section{CORRESPONDENCIA:}

Enrique Otoya

enrique.otoya.d@gmail.com

Fecha de recepción: 04-09-202I.

Fecha de aceptación: 02-I|-202I.

Fuente de financiamiento: por el autor.

Conflictos de interés: ninguno, según declaración del autor. 